\title{
Review
}

\section{Conservative treatment of the neuropathic bladder in spinal cord injured patients}

\author{
JJ Wyndaele*,1, H Madersbacher ${ }^{2}$ and A Kovindha ${ }^{3}$ \\ ${ }^{1}$ Department of Urology, University Antwerpen, Belgium; ${ }^{2}$ The Neuro-urology Unit, University Hospital, Innsbruck, \\ Austria; ${ }^{3}$ Department of Rehabilitation Medicine, Chiang Mai University, Thailand
}

\begin{abstract}
Different conservative treatment modalities for the lower urinary tract dysfunction in patients with spinal cord lesion are reviewed. Conservative treatment is still the mainstay of the urological management in these patients. Growing experience has changed the classical approach. Spontaneous voiding with and without triggered voiding and/or bladder expression has proven to be less safe except in well defined patients with regular urological follow-up. Nowadays, intermittent catheterisation and self catheterisation with and without bladder relaxants are accepted as the methods of choice. Condom catheters are still needed if incontinence persists, while penile clamps have no place in the treatment of patients with spinal cord lesions. Long-term indwelling catheters should be avoided. External electrical stimulation can be used to correct the neurogenic dysfunction by neuromodulation and/or to induce a direct therapeutic response in the lower urinary tract.
\end{abstract}

Spinal Cord (2001) 39, 294-300

Keywords: spinal cord lesion; neuropathic bladder; conservative treatment; urology

\section{Introduction}

The history of the modern treatment of the neuropathic bladder in spinal cord lesion (SCL) patients is relatively short but is soon to celebrate its 50th birthday. This only applies, however, if we accept that the major findings published by Bors, ${ }^{1}$ Comarr, ${ }^{2}$ Guttmann $^{3}$ and others in the 1950s and 1960s gave birth to a better understanding and a better approach of SCL medicine as a whole and to the urological part it plays more specifically.

However, 50 years on, we are still faced with some of the same problems which our distinguished teachers experienced. Last year a survey was published in European Urology ${ }^{4}$ which was quite a shock to an attentive reader. The data given in this survey showed that the acute care of the bladder in SCL patients can still give rise to a frightening number of complications, some of which are difficult to treat. Also, other aspects of neuropathic bladder management can still be improved. Despite 50 years of intensively promoting the correct acute urinary treatment after spinal cord lesions, studies similar to the one previously mentioned make us aware of how far the clinical reality can stand away from the ideal situation created in

*Correspondence: JJ Wyndaele, Department of Urology, UZA, 10 Wilrijkstraat, B 2650 Edegem, Belgium ideal settings. It invites reflection and urges more continuous action. It makes us realize that work is seldom ever finished, but needs to be continued, repeated and improved.

The important clinical and scientific work carried out in the second half of the twentieth century has produced a major evolution in bladder management.

This study presents an updated overview of what conservative management of the neuropathic bladder can be and should be today. How we should treat a SCL patient nowadays has become very different compared to the teaching at the beginning of our careers.

\section{Treatment modalities}

Decisions on treatment should depend on an accurate diagnosis of what type of neurologic dysfunction is present in a specific patient. Not only the bladder activity but also the coordination with the bladder neck and the striated external sphincter needs to be studied and documented.

However, the urological treatment starts from the admission of a patient after trauma or lesion. During the acute phase of spinal shock, correct bladder drainage by intermittent catheterization (IC) is the preferred treatment if a large urine output occurs. A 
suprapubic fine bore cystostomy or even an indwelling catheter (ID) can be used, although the latter is less preferable in male patients. Without doubt, it is best not to leave a catheter in place for long periods of time. If an indwelling catheter is judged necessary, complications can be limited by strict catheter care. The importance of a team approach to urinary bladder management has also been stressed. ${ }^{5}$

How should IC be carried out in the hospital? Two main techniques are currently described: (1) a sterile and (2) a clean technique. In the sterile, non-touch technique advocated by Guttmann and Frankel ${ }^{6}$ sterile materials are used by a sterile non-touch technique with sterile gloves and forceps. In an intensive care unit, some advocate wearing a mask and a sterile gown as well. In some centres patients are taught to catheterize themselves within a few weeks after the lesion. Self-catheterization (ISC) has proven to be practical in patients with good hand function and it makes cross infections in a ward almost completely disappear. $^{7}$

After a spinal shock resolves, the new neurologic and functional situation of the lower urinary tract (LUT) gradually becomes clear. Clinical neurological examination gives useful information, and to a certain degree reflects the LUT function. Urodynamic studies are necessary for a proper evaluation of the function of different parts of the LUT and their interaction. ${ }^{8}$ Cystometry and other urodynamic tests should be repeated to follow the LUT evolution, to evaluate the effect of treatment, and to discover, as early as possible, aspects of an unbalanced bladder with risks for the upper urinary tract. ${ }^{9}$

Madersbacher et $a l^{10}$ have standardized the various types of neuropathic bladder dysfunction. Damage to the innervation of the LUT mostly affects the detrusor and the sphincter. Both can become either hyperreflexic or areflexic. Clinically the combination corre- sponds with mainly four types: (1) hyperreflexic detrusor with hyperreflexic sphincter which almost always means detrusor-sphincter dyssynergia; (2) hyperreflexic detrusor with hypo-/areflexic sphincter; (3) hypo-/areflexic detrusor with hyperreflexic sphincter and (4) hypo-/areflexic detrusor with hypo-/ areflexic sphincter. Table 1 gives an overview of each type with the possible treatment modalities. The following are critical evaluations of each treatment type.

\section{Triggered reflex voiding}

The importance of triggered reflex voiding has decreased considerably in the era of clean intermittent catheterization (CIC). Nevertheless it is still in use. According to the ICS committee on standardisation of terminology, bladder reflex triggering comprises various manoeuvres performed by the patient or by the carer in order to elicit reflex detrusor contractions by exteroceptive stimuli. ${ }^{11}$ The aim of regular triggered voiding is to regain control over the reflex bladder, ie, whenever a patient wants to void, the bladder is triggered to contraction. However, this type of voiding has many pitfalls and the clinical outcome is not very good. In suprasacral lesions a reflex bladder normally develops. Bladder emptying via this triggered sacral reflex is unphysiological: it comprises $\mathrm{C}$-fibre activation, bladder contraction is involuntary and not sustained, detrusor-striated sphincter dyssynergia or detrusor-bladder neck dyssynergia is present in over $90 \%$ of patients, and autonomic dysreflexia can be associated. In addition, few patients have a balanced voiding, ie a residual urine less than $25 \%$ of the functional bladder capacity or with less than $100 \mathrm{ml} .{ }^{12}$

There are many ways to perform triggering. ${ }^{13}$ Some achieve a prompt voiding after rhythmic suprapubic percussions while others provoke strong spasticity of

Table 1 Types of neurologic dysfunction of the lower urinary tract after spinal cord lesion, the urodynamic data and treatment modalities

Types of neurologic bladder and

sphincter dysfunction

Hyperreflexia of detrusor and sphincter.

Former: Upper motor neuron bladder

Former: Lower motor neuron bladder

Detrusor areflexia with sphincter hyperreflexia

Detrusor hyperreflexia with sphincter areflexia
Urodynamic data

Detrusor involuntary contractions + sphincter dyssynergia.

Reflex incontinence

Residual urine

No activity in detrusor or sphincter

Stress incontinence

Residual urine

Overflow incontinence

Urinary retention

Reflex incontinence and stress incontinence
Possible treatment modalities

Intermittent (self) catheterisation Bladder relaxing drugs

Electrotherapy

External appliances

Triggered voiding

Indwelling catheter

External appliances

Bladder expression

Drugs

Surgery

Intermittent selfcatheterisation

Indwelling catheter

Bladder relaxant drugs

Electrotherapy

External appliances

Surgery 
the pelvic floor and the external striated sphincter, which prevents expulsion of urine for some time or makes the outflow interrupted. Some patients can improve the contraction by continuing tapping but others have a better voiding when tapping is stopped after 7-8 percussions. Other manipulations such as thigh scratching, touching the penile skin, pulling the pubic hair, ano-rectal manipulation are successful in some patients. ${ }^{12}$ Low and Donovan ${ }^{14}$ have proved that stretching the anal sphincter helps overcome urethral sphincter spasticity.

It is well known that the pressure development in the bladder, the strength of the detrusor contraction and the duration of the high detrusor contraction are crucial for the long-term outcome of the urinary tract. Reflex voiding is based on an unphysiological sacral reflex and its use by triggering the reflex several times a day is a potentially dangerous procedure with a limited role in spinal cord injury patients. Deterioration of bladder function and form, hydronephrosis and renal impairment have been described. ${ }^{15}$ Therefore, urodynamics should be performed in all patients starting or continuing with triggered voiding.

Continence is a problem in many patients on triggered voiding due to spontaneous contractions of the bladder between triggering episodes. Bladder relaxing drugs may be tried but external appliances are often necessary. If the outflow obstruction remains a problem surgery, eg sphincterotomy, to relieve the obstruction may have a role. Eighty per cent of patients who develop reflex voiding and require an external urine collecting device for control of incontinence have chronic or recurrent bacteriuria. ${ }^{16}$

The working group on Conservative Management in the Neuropathic Patient of the 1st International Consultation on Incontinence, have summarized indications and contraindications. ${ }^{10}$ The indication must be based on (video-)urodynamics to find out whether the urodynamic situation is safe: one should elicit an adequate detrusor contraction with bladder pressures less than $70-80 \mathrm{~cm} \mathrm{H}_{2} \mathrm{O}$ in males and $40-$ $60 \mathrm{~cm} \mathrm{H}_{2} \mathrm{O}$ in females resulting in balanced voiding. In addition, triggered voiding can be recommended for patients after sphincterotomy/bladder neck incision, in order to support or improve spontaneous reflex voiding. The patient who achieves balanced voiding by this method, and when a good solution can be found for reflex incontinence, is the best candidate.

Triggered voiding is not recommended if there is no adequate detrusor contraction (too low, too high, too short or too long), if it results in unbalanced voiding, if vesico-uretero-renal reflux is present, if in the male a reflux in the seminal vesicules or in the vas is present, if there is uncontrollable autonomic dysreflexia or if recurrent urinary tract infection persists.

\section{Bladder expression}

Bladder expression comprises various manoeuvres aiming at increasing intravesical pressure in order to enable or to facilitate bladder emptying. Bladder expression has been recommended for a long time for patients with so-called lower motor neuron lesions, resulting in a combination of an underactive detrusor with an underactive sphincter or with an incompetent urethral closure mechanism of other origin.

The most commonly used manoeuvres are the Valsalva (abdominal straining) and the Credé (manual compression of the lower abdomen) manoeuvre. Clinical experience has shown that by bladder expression many patients are able to empty their bladders, albeit mostly incompletely. Urodynamics/ videourodynamics have demonstrated that despite high intravesical pressures, created by these manoeuvres, the urinary flow may be very poor and residual urine present. Difficulty in emptying the bladder in this way may be due to contraction of the external sphincter and/or to an inability to open the bladder neck. ${ }^{17}$ Particularly in patients with complete flaccid paralysis of the musculature of the pelvic floor, these manoeuvres induce a mechanical obstruction at the level of the striated external sphincter. Voiding-cystourethrogram shows a typical bending, deformation and narrowing of the membranous urethra at the level of the pelvic floor induced by downward pushing. ${ }^{18}$ This narrowing cannot be recognised by a retrograde urethrogram, nor felt by catheterisation, nor is it visible endoscopically. Clarke and Thomas ${ }^{19}$ studied static urethral pressure profiles in paraplegics with an acontractile bladder and showed that the urethral pressure in all flaccid male paraplegics investigated was much higher at the external sphincter than at the bladder neck. It was largely abolished by alpha-blockers. Their conclusion was that in flaccid male paraplegics the major component of urethral resistance is a constant, adrenergically innervated muscular resistance in the distal intrinsic urethral sphincter. Alpha-blockers, if effective, usually increase urinary stress incontinence. $^{20}$

With increasing time, more than $40 \%$ of the patients show demonstrable influx into the prostate and the seminal vesicles, and other complications due to the high pressures during the Valsalva or Credé manoeuvre. These unphysiologically high pressures may also cause reflux to the upper urinary tract with all known consequences.

Bladder expression may only be recommended for patients with an underactive detrusor with an underactive or incompetent sphincter mechanism. It must be emphasised that sphincter-hyperreflexia and detrusorsphincter dyssynergia are contraindications for bladder expression. There is one exception. When used in the reflex bladder together with the anal sphincter stretch described by Low and Donovan: ${ }^{14}$ by inserting one or two gloved fingers into the anal canal and then stretching the anal sphincter, the external urethral sphincter relaxes and voiding is then achieved by abdominal straining. Bladder expression is contraindicated if it induces a high intravesical pressure. 
Moreover, an existing vesico-uretero-renal reflux, reflux into the male adnexa, hernias and haemorrhoides as well as urethral pathology and symptomatic UTI's are contraindications.

\section{Condom catheters}

Condom catheters (external catheters) aim at collecting leaking urine into a device. They will prevent spilling of the urine and give better hygienic control, better control of unpleasant odour and a better quality of life. Due to the fact that they are not invasive, they permit prevention of most of the complications related to indwelling catheters. Old versions of the currently used condom catheters are reusable devices fitted rather loosely around the penis. They are still preferred by a few paraplegics who have been accustomed to them for a long time, especially those with a retractile penis. They are also still used in developing countries due to their low cost.

More modern condom catheters are thin conicalshaped sheaths reinforced at the tip to prevent kinking and twisting. They are made of rubber, latex, silicone or other plastic material. They fit over the shaft of the penis towards the peno-scrotal junction. Their tips are open and connected with the tube of a urinary collecting device.

With a smaller and/or retractile penis and/or abundant pubic fat, difficulties of fixation may occur. To overcome this inconvenience, different sizes of condom catheters and different means of adhesion of the sheath to the penis have been manufactured. The implant of a penile prosthesis may be a possible solution for those with a retractile small penis. In recent years special condoms and special devices allowing urethral catheterisation without removing the condom have been manufactured. However, the clinical experience is still limited.

Although the advantages of condom catheters over indwelling catheters and incontinence pads are evident, they can lead to problems and complications, sometimes severe. Newman and Price ${ }^{21}$ found bacteriuria in more than $50 \%$ of patients using a condom catheter. One of the factors correlated with increased risk for UTI was the changing of condoms less than daily. ${ }^{22}$

Lesions of the penis can be secondary to mechanical damage to the skin from an excessively tight condom worn for a prolonged time. Preventive measures are to discontinue the use of the condom during part of the day, especially at night, and to replace them with bottle urinals or other appliances. Another common cause of skin lesions is allergy to the material of the condom, usually to latex. This kind of allergy is well documented in patients with long-term use of latex products, especially in myelomeningocele patients and in patients with a spinal cord lesion. In severe cases, it has caused life threatening episodes of intraoperative cardiovascular collapse. ${ }^{23}$ In the case of latex-allergy the best prevention is to use a latex-free (silicone, vinyl, etc.) condom catheter.
In conclusion, a condom catheter may be indicated in male spinal cord lesioned patients with urinary incontinence provided that they have no penile lesion and that intravesical pressures during storage and voiding are urodynamically safe. No absolute contraindications for such appliances seem to exist. ${ }^{10}$

\section{Penile clamps}

These are not recommended for patients with neuropathic voiding dysfunction, due to the danger of skin and urethral lesions.

\section{Electrical stimulation}

The aim of electrical stimulation is to modulate LUT dysfunction or to directly induce a therapeutic response.

Non-invasive electrical therapy for neurogenic LUT dysfunction comprises mainly neuromodulation for detrusor hyperreflexia and intravesical electrostimulation for the hyposensitive and hypocontractile detrusor.

There are two main effects of therapeutic electrical stimulation of pudendal nerves: (1) sphincter activation and (2) bladder inhibition. Bladder inhibition can be achieved in various ways: by direct stimulation of the sacral roots or of the pudendal nerve itself or indirectly by stimulating its branches either transcutaneously via the dorsal penile/clitoridal nerve, via the anal or vaginal mucosa activating its branches in the pelvic floor musculature or by stimulation of segmental afferent nerves. The general principle is to activate artificially normal inhibitory reflexes.

In 1986 Vodusek and Light $^{24}$ described strong detrusor inhibition induced by stimulation of pudendal nerve afferents and demonstrated the effects in patients suffering from detrusor hyperreflexia after suprasacral spinal cord injury. The complex nature of the central nervous control of micturition implies numerous possible explanations of the therapeutic effects. The inhibitory effect on the urinary bladder is mediated by activation of pudendal afferents resulting in hypogastric nerve stimulation, direct inhibition of the pelvic nerve outflow and also by cortical inhibition as shown by Jiang ${ }^{25}$ and by Oliver et $a .^{26}$

For detrusor inhibition short-term maximal electrical stimulation is applied in the clinic or later as a home treatment programme. Short-term stimulation usually consists of a series of 20-30 min sessions, once or twice a day. To elicit optimum bladder inhibition, a stimulation frequency of $5-10 \mathrm{~Hz}$ and a pulse duration of $0.2 \mathrm{~ms}$ have been found most efficient, with intensity below the pain-threshold if sensation is present. Clinical experience has shown that this type of stimulation has a certain re-education or carry-over effect, which implies that the effect of stimulation has a longer duration than the stimulation itself. This might be due to long-term potentiation for which Jiang $^{18}$ has demonstrated a neuropharmacologic basis. The 
efficacy of stimulation, the inhibition effect, is dependent on the proximity of the electrode to the appropriate nerve, the number of nerve branches activated and whether specific branches are stimulated. Direct stimulation of the pudendal nerve is more effective than stimulating its pelvic floor branches via the vaginal or anal mucosal surface. According to the findings of Nakamura and Sakurai ${ }^{27}$ the transcutaneous access to the pure sensory, dorsal penile or clitoral nerve, using clip electrodes in the female and ring electrodes in the male would be preferable (transcutaneous electrical nerve stimulation of the pudendal nerve (TENS-P).

If non-invasive electrical neuromodulation fails, invasive sacral neuromodulation should be considered. This will not be discussed in this study.

Intravesical electrostimulation (IVES) is not a new technique. In 1878 the Danish surgeon Saxtorph ${ }^{28}$ recommended IVES to treat patients with urinary retention. In 1899 two surgeons in Vienna, FrankelHochwart and Zuckerkandl, ${ }^{29}$ reported that 'faradisation' intravesically was much more effective than the transcutaneous technique. The method was rediscovered by Katona et $a l^{30}$ in 1959 as a method to treat weak detrusor contractility in children with myelomeningocele. Although several studies reported good results, the procedure has not been generally accepted for a long time. The scepticism was caused possibly by a lack of theoretical background and little clinical documentation.

In animal experiments Ebner et $a l^{31}$ proved that IVES induced reproducible detrusor contractions at volumes below micturition threshold. The contractions elicited were reflex responses. From the characteristics of the afferent discharge measured on the posterior sacral roots during IVES these authors proved that this technique involves an artificial activation of bladder mechanoreceptor afferents of the A-delta type, known to be responsible for the normal micturition reflex. The increase of the afferent input also increases the efferent output to the bladder. Prerequisites for successful IVES are incomplete nerve lesions where at least some afferents have remained intact, the mechanoreceptors must be intact, the detrusor must still be able to contract and it needs a cerebral cortex able to perceive afferent stimuli.

Indications can be found amongst children with congenital neurogenic detrusor dysfunction and amongst adults with incomplete spinal or peripheral neurological lesion. The advantage of IVES is that there are no side effects. The disadvantages are that IVES needs the services of specialized personnel, although home treatment is possible, but this is very time consuming. Moreover, there is no simple investigation of predictive value, but the presence of cortical evoked potentials with electrical stimulation of the bladder neck, indicating functioning afferent fibres from the bladder to the cortex, is helpful. IVES on a more chronic basis is necessary in $70 \%$ of patients with neurogenic bladder dysfunction. ${ }^{32}$

\section{Intermittent catheterisation}

The use of catheters for bladder emptying dates back many thousands of years. Intermittent catheterisation (IC) and self-catheterisation (ISC) have become more acceptable over the last 40 years.

The general aims of IC and ISC are to empty the bladder and to prevent bladder overdistension in order to avoid complications and to improve urological conditions. The main indication is an inability to empty the bladder adequately due to conditions which may be neuropathic.

Many studies show good results both in the acute and in the long-term stages. Therefore, IC and ISC are nowadays the main choice of methods recommended for the management of neurologic bladder dysfunction. 10

Many types of catheters can be used. Choices depend on availability and cost. In developing countries where IC has become very much accepted, limitation of resources are still the main problem. ${ }^{10}$ Also the material has an important role ie, silicone or plastic is needed if latex allergy exists. Silicone, rubber, glass and stainless steel are alternatives if the catheter is reused. ${ }^{33}$ Size $10-14 \mathrm{Fr}$ for males and 14-16 for females are preferable but bigger sizes may be necessary when urine is very cloudy and after bladder augmentation.

If the decision is made to treat with IC or ISC, the patient's education is of utmost importance. They should learn the correct technique and should have a chance to discuss with their physicians or nurses when specific questions and problems are encountered. The basic principles of good catheterisation should be used. Catheterisation must be atraumatic and with necessary precautions to prevent infection.

To be atraumatic the catheterisation has to be carried out with a normal size catheter, with a good quantity and type of lubricant and with a good and gentle handling of the catheter. For the latter, sufficient hand function is necessary if ISC is recommended.

To prevent infection the meatus should be cleaned and a clean and preferably sterile or resterilized catheter should be used. The catheter must be carefully handled in order to keep it clean during the introduction into the bladder. The bladder has to be emptied completely. A frequency of catheterisation between 4-6 times a day has proved to be optimal if the patient has normal drinking habits.

ISC in the chronic stage after spinal cord lesion is carried out in a clean, non-sterile way which is applicable in most places.

IC and ISC are very good techniques but the patient must be aware that complications can occur. Urinary tract infection in patients on chronic ISC has a prevalence of 13.6 infections per 1000 patient-days. ${ }^{34}$ Genito-urinary complications such as urethritis or epididymo-orchitis are rare. ${ }^{35}$ Prostatitis is underestimated and probably has a prevalence around 5\% to $18 \%{ }^{36}$ Urethral bleeding is frequent. False passage, 
meatitis and meatal stenosis are rare. Urethral strictures in male patients increase in prevalence with longer follow-up. ${ }^{37}$

IC and ISC seldom stand alone. Their use together with drugs can overcome incontinence and recurrent infections.

It is mandatory to have a regular follow-up and this includes urodynamic investigation even if the patient is continent and infection-free, because the neuropathy can silently cause LUT deformities with impact on the upper urinary tract.

IS and ISC are not possible in all patients. Some have poor hand function and no other person to perform the catheterisation. Unwillingness of the patient, high cost, lack of knowledge from carers, persistent incontinence, generally poor condition or difficulty to reach the meatus can be reasons why ISC is not practical. In many cases these problems may be overcome with good treatment and education. However, in some cases an indwelling catheter will be used.

\section{Indwelling catheters}

Transurethral and suprapubic catheters have been used for a long time. The dangers of the techniques have been well documented and the complications are well known.

If they are used it is very important to apply good rules of indwelling catheter management. A catheter size of $12-14 \mathrm{Fr}$ is recommended to avoid blocking of the urethral lumen and still permitting a good outflow of urine. The catheter should be properly introduced with the balloon positioned in the bladder: it is important to be especially careful in the presence of a spastic sphincter. The outflow should be controlled regularly to avoid overdistension of the bladder. The catheter should be changed regularly several times a week in an acute situation; every 10 days in a more chronic clinical setting and every 4-6 weeks in a chronic patient who has little complications. ${ }^{38}$

Anticholinergic drugs may be important in patients with bladder hyperreflexia to prevent the development of small contracted bladder. Antibacterial drugs should not be used to prevent or treat an asymptomatic infection of the urine. ${ }^{39}$ With an indwelling catheter the prevalence of infection is $100 \%$ if the catheter is used for more than 2 weeks. ${ }^{40}$ If symptomatic infection occurs, medical treatment is necessary.

Suprapubic fine bore catheters may be preferable especially in the male patient as it leaves the urethra open. ${ }^{10}$ But urinary tract infection develops in all patients if the suprapubic catheter is used for more than 5-7 weeks. ${ }^{4}$

There is no general agreement on clamping the catheter for bladder training. It was aimed to prevent a contracted bladder, to condition a time-related reflex pattern and to increase patient awareness of bladder function. $^{42}$ However, this technique is not well accepted in neuropathic patients due to the possibility of infection, unawareness of overdistension during the acute stage and leakage around the catheter in chronic spastic bladder. In cases of severe incontinence and where unsuccessfully treated with drugs, a continuous outflow is perhaps the only solution. If clamping is considered it is necessary to avoid bladder spasticity with drugs or other treatment. Complications are frequent. The permanent catheters can cause acute septic episodes, urethral trauma and bleeding, false passages, strictures, diverticuli and fistuli of the urethra, bladder stones, squamous cell bladder carcinoma, epididymo-orchitis and prostatitis. ${ }^{43,44}$ With good daily care many of these can be avoided. The role of education is most important.

The presence of an indwelling catheter should be known to occupational therapists, physiotherapists, nursing staff and all who care for the hospitalised patient.

\section{Pharmacologic treatment}

Drugs are used for many different indications in patients with LUT neuropathy. Bladder relaxant drugs, bladder afferent blocking drugs and drugs to influence urethral resistance are an important part of treatment. $^{10}$ New drugs are developed on a regular basis to try to overcome shortcomings in specificity, complications and side effects. The topic will not be discussed further in this study.

\section{Conclusion}

The conservative treatment of the neurologic bladder in patients with a spinal cord lesion has seen considerable changes during the last century. Some techniques such as triggered voiding and bladder expression have lost their predominant position. Intermittent catheterisation is now accepted as the method of choice if applicable. The treatment however must be based on a clear diagnosis of the urodynamic function, the patient's personal abilities and wishes. Treatment must first of all aim at keeping the patient alive but can now have a marked effect on the quality of their lives.

\section{References}

1 Bors E. Urologic aspects of rehabilitation in spinal cord injuries. J Amer Med Ass 1951; 146: 225-225.

2 Comarr AE. The practical urological management of the patient with spinal cord injury. Br J Urol 1959; 31: 1-46.

3 Guttmann L. Discussion on the treatment and prognosis of traumatic paraplegia. Proc Roy Soc Med 1947; 40: $219-225$

4 Zermann D et al. Audit of early bladder management complications after spinal cord injury in first-treating hospitals. Eur Urol 2000; 37: 156-160. 
5 Lindan R, Leffler E, Freehafer AA. The team approach to urinary bladder management in spinal cord injury patient: a 26 year retrospective look at the Highland View Urinary Catheter Care Team. Paraplegia 1990; 28: $314-317$

6 Guttmann L, Frankel H. The value of intermittent catheterization in the early management of traumatic paraplegia and tetraplegia. Paraplegia 1966; 4: 63-83.

7 Wyndaele JJ, De Taye N. Early intermittent self catheterization after spinal injury. Paraplegia 1990; 28: $76-80$.

8 Wyndaele JJ. Correlation between clinical neurological data and urodynamic function in spinal cord injured patients. Spinal Cord 1997; 35: $213-216$.

9 Wyndaele JJ. A critical review of urodynamic investigations in spinal cord injury patients. Paraplegia 1984; 22: $138-144$

10 Madersbacher et al. Conservative management in the neuropathic patient. In: Abrams P, Khoury S, Wein A (eds). Incontinence. Health publications, 1999, pp 775812.

11 Andersen JT et al. Lower urinary tract rehabilitation techniques: seventh report on the standardisation of terminology of lower urinary tract function. Neurourol Urodyn 1992; 11: $593-603$.

12 Opitz JL. Bladder retraining: an organised program. Mayo Clin Proc 1976; 51: $367-372$.

13 Rossier A, Bors E. Detrusor response to perineal and rectal stimulation in patients with spinal cord injuries. Urol Int 1964; 10: $181-190$.

14 Low AI, Donovan WD. The use and mechanism of anal sphincter stretch in the reflex bladder. Br J Urol 1981; 53: $430-432$.

15 Yokoyama $\mathrm{O}$ et al. Morphological and functional factors predicting bladder deterioration after spinal cord injury. J Urol 1996; 155: $271-274$

16 Lloyd LK, Kuhlemeier KV, Stover SL. Initial bladder management in spinal cord injury: does it make a difference? J Urol 1986; 135: 523-526.

17 Barbalias GA, Klauber GT, Blaivas JG. Critical evaluation of the Crede manoeuvre: a urodynamic study of 207 patients. J Urol 1983; 130: $702-703$.

18 Madersbacher $\mathrm{H}$. The neuropathic urethra: urethrogram and pathophysiologic aspects. Eur Urol 1977; 3: $321-$ 322.

19 Clarke SJ, Thomas DG. Characteristics of the urethral pressure profile in flaccid male paraplegics. $\mathrm{Br} J \mathrm{Urol}$ 1981; 53: $157-161$

20 Scott MB, Marrow JW. Phenoxybenzamine in neurogenic bladder dysfunction after spinal cord injury. I. Voiding dysfunction. J Urol 1978; 119: 480-482.

21 Newman E, Price M. External catheters: hazards and benefits of their use by men with spinal cord lesions. Arch Phys Med Rehabil 1985; 66: 310-313.

22 Waites KB, Canupp KC, DeVivo ML. Epidemiology and risk factors for urinary tract infection following spinal cord injury. Arch Phys Med Rehabil 1993; 74: 691-695.

23 Shenot P, Rivas DA, Kalman DD, Staas Jr WE. Latex allergy manifested in urological surgery and care of adult spinal cord injured patients. Arch Phys Med Rehabil 1994; 75: $1263-1265$.

24 Vodusek DB, Light JK, Libby JM. Detrusor inhibition induced by stimulation of pudendal nerve afferents. Neuourol Urodyn 1986; 5: 381-389.
25 Jiang $\mathrm{CH}$. Modulation of the micturition reflex pathway by electrical stimulation: An experimental study in the rat. Neurourol Urodyn 1998; 17: 543-553.

26 Oliver SA et al. What does neuromodulation do for the sensations of urinary urge and urgency. Neurourol Urodyn 1999; 18, 403 (Proceedings of the International Continence Society 1999).

27 Nakamura M, Sakurai T. Bladder inhibition by penile electrical stimulation. Br J Urol 1984; 56: 413-415.

28 Saxtorph MH. Stricture urethrae-Fistula perineiRetentio urinae. Clinisk Chirurgi. Copenhagen: Gyldendalske Forlag 1878: $265-280$.

29 Frankl-Hochwart LV, Zuckerkandl O. Die nervösen Erkrankungen der Blase. In: Spezille Pathologie und Therapie. Ed van Nothnagel. Wien: Holder, 1989.

30 Katona F, Beyo L, Lang J. Über intraluminäre Elektrotherapie von verschiedenen paralytischen Zuständen des gastrointestinalen Traktes mit Quadrangulärstrom. Zbl Chir 1959; 84: 929-933.

31 Ebner A, Jiang CH, Lindström S. Intravesical electrical stimulation. An experimental analysis of the mechanisms of action. J Urol 1992; 148: 920 - 924.

32 Madersbacher $\mathrm{H}$ et al. Rehabilitation of micturition in adults with incomplete spinal cord lesions by intravesical electrotherapy. Neurourol Urodyn 1987; 6: 230-232.

33 Kovindha A, Na W, Madersbacher H. Radiologic abnormalities in spinal cord injured men using clean intermittent catheterization with a re-usable silicone catheter in developing country. Abstract book 39 annual scientific meeting IMSOP Sidney Australia 2-5 November 2000. Abstract 86, p 110.

34 Perkash I, Giroux J. Clean intermittent catheterization in spinal cord injury patients: a follow-up study. $J$ Urol 1993; 149: 1068 - 1071.

35 Maynard FM, Glass J. Management of the neuropathic bladder by clean intermittent catheterization: a 5 year outcome. Paraplegia 1987; 25: $106-110$

36 Wyndaele JJ. Chronic prostatitis in spinal cord injury patients. Paraplegia 1985; 23: $164-169$.

37 Wyndaele JJ, Maes D. Clean intermittent selfcatheterization: a 12 year follow-up. J Urol 1990; 143: 906-908.

38 Macdiarmid S et al. Urological neurology and urodynamics. Management of spinal cord injured patients by indwelling suprapubic catheterization. J Urol 1995; 154: $492-494$

39 Kunin CM, Steele C. Culture of the surfaces of urinary catheters to sample urethral flora and study the effect of antimicrobial therapy. J Clin Microbiol 1985; 21: $902-$ 908.

40 Stamm WE. Guidelines for prevention of catheter associated urinary tract infections. Annals Int Med 1975; 82: $386-390$.

41 Hackler RH. Long-term suprapubic cystostomy drainage in spinal cord injury patients. Br J Urol 1982; 54: $120-$ 121.

42 Yarnell SK, Checkles NS. Intermittent catheterization: longterm follow-up. Arch Phys Med Rehab 1978; 59: $491-496$

43 Jacobs SC, Kaufman JM. Complications of permanent bladder catheter drainage in spinal cord injury patients. $J$ Urol 1978; 119: $740-741$.

44 Trop CS, Bennett CJ. Complication from long-term indwelling foley catheters in female patients with neurogenic bladder. Semin Urol 1992; 10: 115-120. 